\title{
The Envelope of Ballistic Trajectories and Elliptic Orbits
}

\author{
Eugene Butikov \\ Saint Petersburg State University, Saint Petersburg, Russia
}

\begin{abstract}
Simple geometric derivations are given for the shape of the "safety domain" boundary for the family of Keplerian orbits of equal energy in a central gravitational field and for projectile trajectories in a uniform field. Examples of practical uses of the envelope of the family of orbits are discussed and illustrated by computer simulations. This material is appropriate for physics teachers and undergraduate students studying classical mechanics and orbital motions.
\end{abstract}

Key words: safety domain, Keplerian orbits, family of orbits, foci of the ellipses, envelope of trajectories, minimal shooting speed.

\section{Introduction: The Envelope of Projectile Trajectories}

A famous old problem of classical mechanics concerns projectiles launched over the Earth from a given point with a given velocity whose direction is varied. All the projectiles start from the origin in various directions and move under the uniform field of gravity in the absence of the air resistance. This results in a family of parabolic trajectories (see Fig. 1) whose envelope defines the border of the so-called "safe domain." It is well known (since the work of Torricelli ${ }^{1}$ back in 1644) that the envelope of these trajectories is a parabola (or a paraboloid of rotation about the vertical axis in three dimensions). This boundary separates the points in space that can be reached by a projectile from those that are out of reach from the given initial point for the given initial speed.

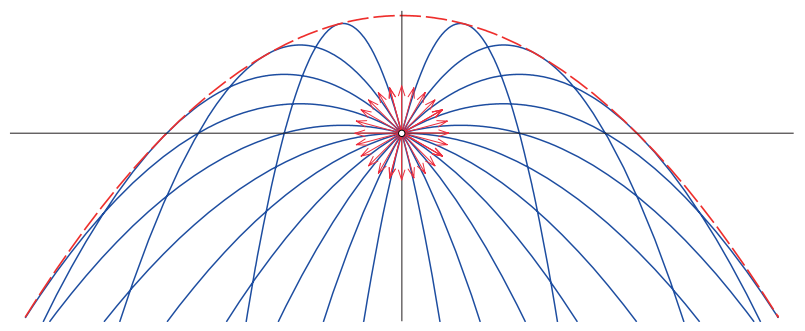

Figure 1: Family of parabolic trajectories traced by projectiles launched from one point in various directions with equal initial speeds, and their envelope (dashed line).

Many different ways for deriving the equation of the envelope are discussed in the literature. It is scarcely possible to reference here all relevant papers. In particular, the problem of the safe domain in a uniform field is treated in Ref. [1], where successive launches with varied initial angle are considered, as well as simultaneous firing in all directions. With some recent articles and books [2]-[5] it is possible to trace back many previous contributions to the issue.

\footnotetext{
${ }^{1}$ An italian physicist and mathematician Evangelista Torricelli (1608-1647), best known for his invention of the barometer, studied a lot about projectiles. "Perhaps his most notable achievement in the field of projectiles was to establish for the first time the idea of an envelope: projectiles sent out at the same point and the same speed in all directions trace out parabolas which are all tangent to a common paraboloid. This envelope became known as the parabola di sicurezza." Philip J. Robinson, The Mathematical Gazette, Vol. 78, No. 481 (Mar., 1994), pp. 37-47.
} 
However, these trajectories are parabolas only approximately, when the initial speed is sufficiently small, and when they are confined to a region within which the gravitational field is nearly uniform (the approximation of the "flat Earth"). Actually, the projectiles move along portions of ellipses whose remote common focus is located at the Earth's center. The greater the initial speed, the greater the deviations of real trajectories from parabolas. Hence we can ask a natural question: what is the real (exact) shape of the envelope of these trajectories?

More generally, let us imagine a rocket, launched from the Earth, rising vertically, and at the highest point of its flight exploding into many fragments (point masses) that fly off in all directions with equal speeds relative to the non-rotating geocentric frame of reference. In the absence of air resistance, the further motion of the fragments occurs solely under the action of Earth's gravity, whose magnitude falls off as the inverse square of the distance from the center of the Earth. If the initial speed is large enough, some of the fragments become satellites, orbiting along various elliptic Keplerian paths. If some of the ellipses cross the surface of the Earth, these trajectories are portions of such ellipses.

If the initial speed of the fragments is small, a portion of such an ellipse above the ground is approximately a parabola. This parabolic shape we usually assign to the trajectory of a projectile in the approximation of the "flat Earth" (that is, in the nearly uniform gravitational field) and in the absence of air resistance.

In any case, all the trajectories are confined to a particular spatial region, if the initial speed of the fragments is smaller than the escape velocity. The boundary of this region is an axially symmetric surface whose axis of symmetry passes through the center of the Earth and the point at which the explosion occurs. What is this surface? What is the shape of the envelope of all elliptic orbits of the family? This question may be of interest to physics teachers and undergraduate students studying classical mechanics and orbital motion.

\section{The Family of Elliptic Orbits}

Several orbits of such families and their envelopes are shown in Fig. 2. These figures and those that follow were generated with the help of one of the interactive simulation programs of the software package [6] developed by the author. We note that the program does not "know" anything about Kepler's laws or analytical solutions; it simply integrates numerically the differential equations of motion under the central gravitational force of the Earth. For the family of orbits in Fig. 2(a), the initial speed $v_{0}$ of the satellites is smaller in magnitude than the "circular velocity" $v_{\text {circ }}$ (the velocity of a satellite in a circular orbit that passes through the initial position), while in Fig. 2(b), $v_{0}$ is greater than $v_{\text {circ }}$.

One focus, at the center of the Earth, is common to all elliptic orbits. The second focus, for the family of orbits traced by satellites launched from a given point with a given initial speed, always lies on a given circle whose center is located at the common starting point. These circles are shown by dashed lines in Fig. 2. The radius of this circle equals the distance between the initial position and the highest point reached by the fragment that is fired vertically upward from the initial position. These results will be proved in the next section.

Since the speeds of the fragments are equal, and since the motion of each fragment begins at the same spatial point, the total energies (per unit mass) of each are equal. The total energy depends uniquely on the major axis of the elliptic orbit in the inverse-square central field (see, for example, Refs. [7]-[9]). Therefore, the major axes of all the orbits are also equal, and, according to Kepler's third law, the periods of revolution of all fragments are equal. That is, all of the fragments whose orbits do not intersect the Earth's surface return to the initial position simultaneously.

A detailed analysis (see the next section) shows that the boundary of the safe domain is a surface of revolution of an ellipse (spheroid) whose foci are at the center of the Earth and at the initial position. The dimensions and eccentricity of the spheroid are determined by the position of the initial point and by the initial speed of the fragments. We will give a rigorous proof and a derivation of the parameters of this bounding surface, relying on the geometrical properties of Keplerian orbits.

For the limiting case of small initial speed $v_{0}$ of the fragments (much smaller than the circular velocity $v_{\text {circ }}$ for the starting point), this bounding surface shrinks into a very narrow (degenerate) ellipsoid spanned across the foci located at the initial point and the center of the Earth. The portion of this ellipsoid near the starting point can be approximated by the familiar envelope of the parabolic trajectories in the uniform gravitational field. 

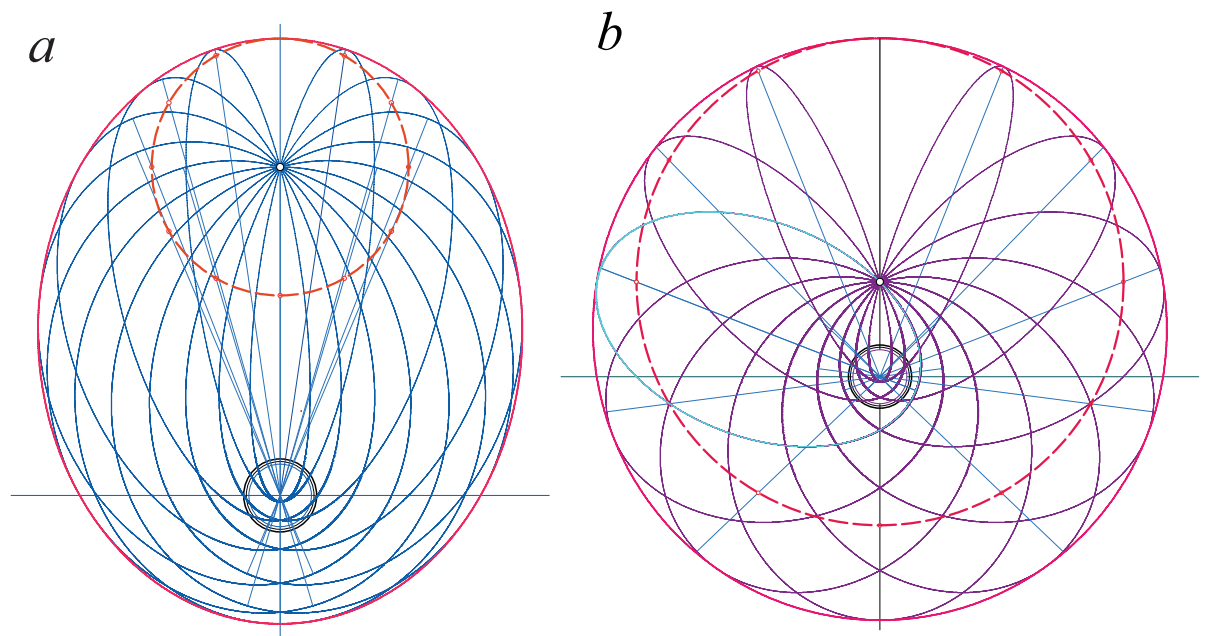

Figure 2: Families of elliptic orbits traced by fragments launched from one point over the Earth in various directions with equal speeds, and the envelope of orbits. Thin straight lines show major axes of the ellipses; dashed circle centered at the initial point is the locus of their second foci. (a) - initial velocity $v_{0}=$ $0.75 v_{\text {circ }},(\mathrm{b})-v_{0}=1.20 v_{\text {circ }}$.

\section{The Shape of the Envelope}

Below we give a simple geometrical proof of the fact that the bounding surface for the family of elliptic orbits with equal energies and a common initial point is a spheroid, generated by the rotation of an ellipse about the axis of symmetry $Z N$ (see Fig. 3). One of the foci of this ellipse is located at the center of the Earth $E$, and the other at the initial point $S$.

The fragment whose initial velocity (velocity at point $S$ in Fig. 3) is directed upward rises directly to the highest point $Z$ located at a distance $r_{\max }$ from the Earth's center. Then it falls toward the Earth along the same line. The trajectory of this fragment is a portion of the rectilinear segment joining the highest point $Z$ with the center of the Earth $E$. We can consider this segment as the limiting case of an infinitely narrow ellipse spanned across points $Z$ and $E$. The foci of this degenerate ellipse lie at the ends $Z$ and $E$ of the segment.

Clearly the highest point $Z$ lies on the bounding surface. The distance $r_{\max }=r_{Z}$ of this point from the Earth's center can be easily calculated by equating the total energy of the fragment at this point $Z$ to the total energy at the initial point $S$, located at the distance $r_{0}$ from the center of force:

$$
\frac{v_{0}^{2}}{2}-\frac{G M}{r_{0}}=-\frac{G M}{r_{\max }}
$$

Here $G$ is the gravitational constant and $M$ is the mass of the Earth. It is convenient to express the gravitational parameter $G M$ of the Earth in Eq. (1) in terms of the escape velocity $v_{\text {esc }}$ for the initial point $S$ (recall that the escape velocity $v_{\text {esc }}=\sqrt{2 G M / r_{0}}=\sqrt{2} v_{\text {circ }}$ is the minimal initial speed that must be imparted to a projectile in order for it to reach infinity):

$$
\frac{1}{r_{\max }}=\frac{1}{r_{0}}\left(1-\frac{v_{0}^{2}}{v_{\mathrm{esc}}^{2}}\right) ; \quad r_{\max }=r_{Z}=\frac{r_{0}}{1-\left(v_{0} / v_{\mathrm{esc}}\right)^{2}}
$$

If the initial velocity equals the circular velocity for the initial point, that is, if $v_{0}=v_{\text {circ }}=\sqrt{G M / r_{0}}$, then Eq. (2) gives $r_{\max }=r_{Z}=2 r_{0}$ : The distance of the highest point $Z$ from the Earth's center is twice the distance $r_{0}$ of the initial point. The distance $r_{\max }=r_{Z}$ tends to infinity if $v_{0} \rightarrow v_{\text {esc }}$.

We can easily find one more point on the desired boundary, namely, the point $N$ on the opposite side (see Fig. 3). It coincides with the apogee (or with the perigee if $v_{0}<v_{\text {circ }}$ ) of the elliptic orbit of the fragment whose initial velocity at $S$ is directed horizontally (is directed transversely to the radius vector). 


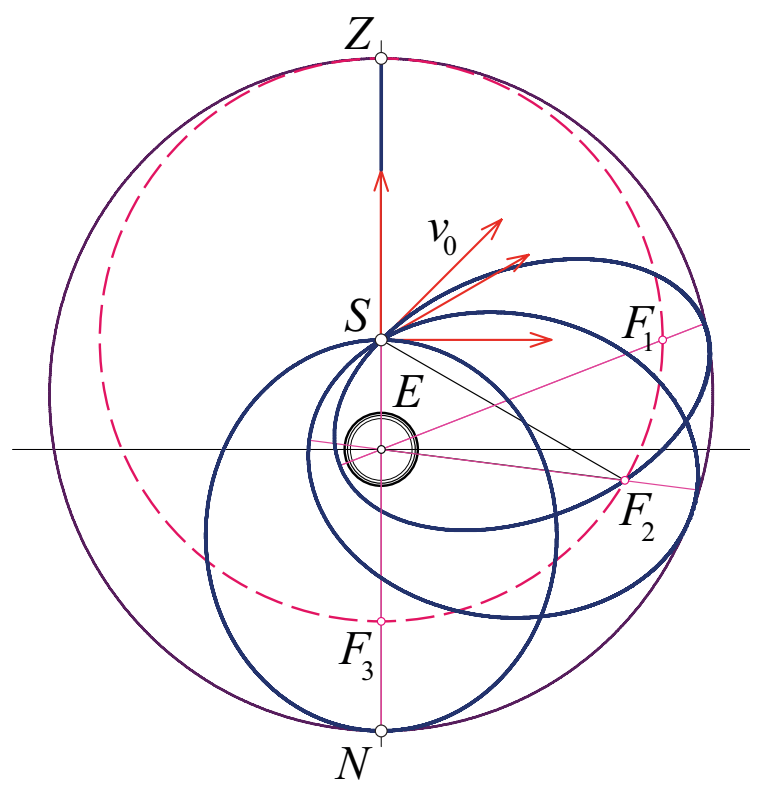

Figure 3: Locus of the second foci of the elliptic orbits (the dashed circle).

The distance $r_{N}$ of this point from the Earth's center can be calculated with the help of the laws of energy conservation and angular momentum conservation $\left(r_{0} v_{0}=r_{A} v_{A}\right)$ :

$$
r_{N}=\frac{r_{0}}{\left(v_{\mathrm{esc}} / v_{0}\right)^{2}-1}
$$

Next we find the locus of the foci of all the orbits of the fragments. All orbits have a common focus at the center of the Earth (Fig. 3), and so the locus of this set is the point $E$. The locus of the set of second foci (points $F_{1}, F_{2}, F_{3}$, and $Z$ in Fig. 3 ) is a circle whose center is located at the initial point $S$, and whose radius is equal to the distance $|S Z|$, measured from $S$ to the most remote point $Z$ (the dashed circle in Fig. 3).

Indeed, for any orbit of the family, the sum of two distances of each point on the orbit from the foci of this orbit equals the major axis of the orbit. The major axes of all the orbits of equal energy constituting the family, as we already mentioned, are equal to one another. Their lengths are equal to the length $r_{\max }$ of the segment $E Z$. We can consider this segment to be the major axis of the degenerate elliptic orbit of the fragment whose initial velocity is directed upwards. All orbits of the family pass through the initial point $S$, and the distance $S E$ from this common point to the focus $E$ for all the orbits is the same and equals $r_{0}$. Consequently, the distance between $S$ and the second focus $F_{1}, F_{2}$, or $F_{3}$ also must be equal for all the orbits. Hence the second foci $F_{1}, F_{2}, F_{3}$ of all orbits of the family lie on the circle whose center is at the initial point $S$ and whose radius equals $|S Z|$ (see the dashed circle in Fig. 3).

Next we prove that the curve whose rotation generates the boundary is an ellipse. The ends of the major axis of this ellipse are located at $Z$ and $N$, and its foci are located at the initial point $S$ and the Earth's center $E$. The shape of the boundary is derived below from the well-known geometric properties of the elliptic trajectories.

We start by considering the following auxiliary construction (Fig. 4): We draw one more circle whose center is at the Earth's center $E$ and whose radius equals $r_{\max }=|E Z|$. This circle passes through $Z$, which lies on the bounding surface.

Now let us consider the problem of finding the orbit of the family that passes through an arbitrary target point $T$ that lies within the second circle just drawn. Choosing $T$ as a center, we draw a third circle tangent to the second circle at point $B$, as shown in Fig. 4. The second focus of the desired orbit passing through $T$ must lie on this circle, because the sum of two distances from the foci again must be equal to $r_{\max }$. And at the same time this second focus of the orbit must lie on the first circle (whose center is at the initial point 

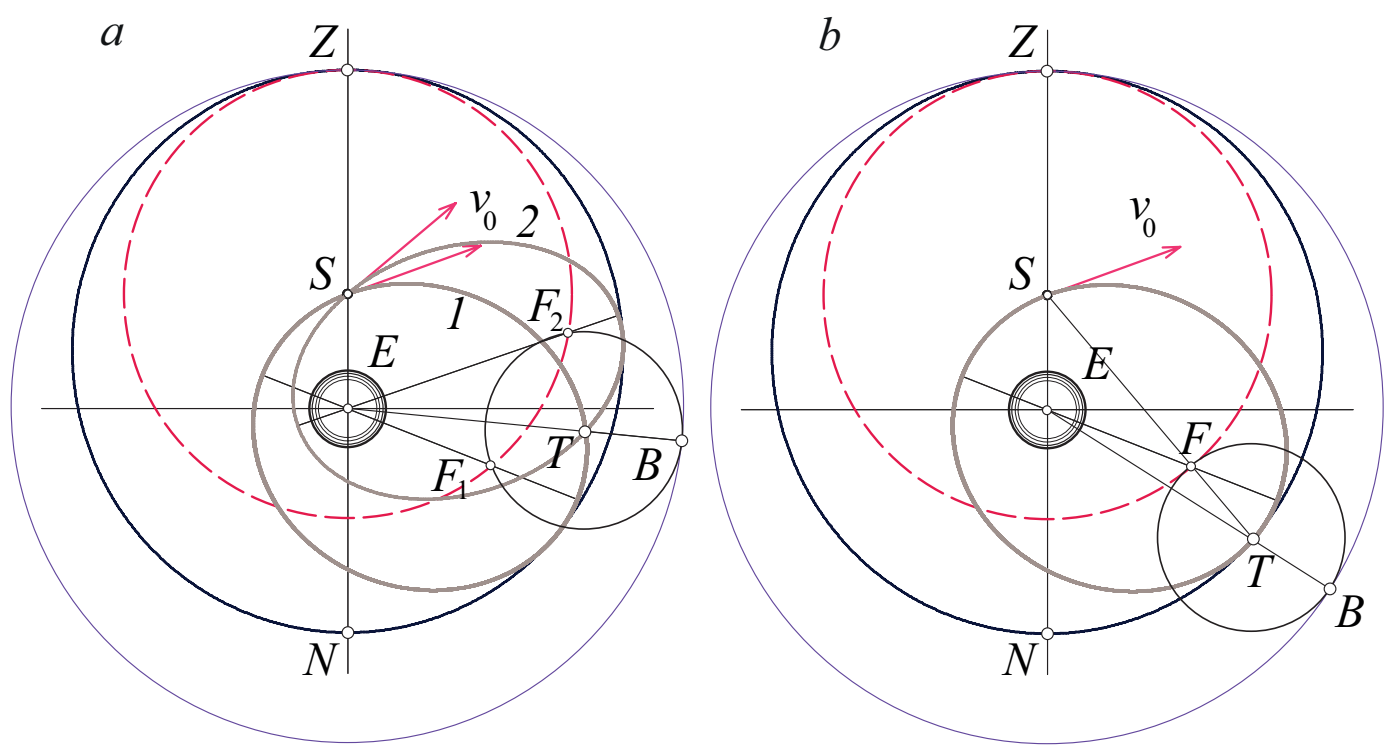

Figure 4: Geometrical proof of elliptical shape of the bounding surface (see text for details).

$S$ and whose radius equals $|S Z|$ ). This circle is shown by the dashed line in Fig. 4. We examine three possibilities:

1. The third circle (whose center is at the target point $T$ ) intersects the first circle (the locus of second foci of the orbits) at two points ( $F_{1}$ and $F_{2}$ in Fig. 4(a)). Then there exist two orbits of the family that pass through the given target point $T$. These orbits are labeled by numbers 1 and 2 in Fig. 4(a). The second foci of these two orbits lie at the two points of intersection $\left(F_{1}\right.$ and $\left.F_{2}\right)$.

2. The third circle has no common points with the first circle. Then no orbit of the family passes through $T$. If follows that the chosen point $T$ lies outside the bounding surface.

3. Lastly, the third circle grazes the first circle, thus having a single common point $F$ with it (see Fig. 4(b)). Then only one orbit of the family passes through the target point $T$. In this case point $T$ must lie on the bounding surface. At point $T$ this single orbit grazes the envelope surface. The foci of this orbit are located at $F$ and the center of the Earth $E$.

We can see from Fig. 4(b) that in the third (grazing) case, the sum of distances from the target point $T$ to the center of the Earth $E$ and to the starting point $S$ equals the radius $r_{\max }$ of the second circle plus the radius $|S Z|$ of the first circle. This sum is independent of the position of point $T$ on the boundary. That is, the sum has equal values for all points of the boundary. Since points $E$ and $S$ are fixed, and since the sum of their distances from $T$ is the same for all $T$, we have proved that the locus of the boundary points for the region occupied by the orbits of the family is an ellipse whose foci are at the center of the Earth $E$ and starting point $S$.

We note that the exploitation of geometrical properties of ellipses allowed us to easily find the envelope of the family of orbits without tedious calculations.

The eccentricity of the bounding ellipse can be found as the ratio of the distance $r_{0}$ between its foci to the major axis $r_{Z}+r_{N}$ (see Fig. 4(b)). Using Eqs. (2) and (3), we find

$$
e=\frac{r_{0}}{r_{Z}+r_{N}}=\frac{v_{\mathrm{esc}}^{2}-v_{0}^{2}}{v_{\mathrm{esc}}^{2}+v_{0}^{2}} .
$$

For a target lying on the bounding surface (see Fig. 4(b)) there exists a unique trajectory of the family of elliptic orbits with a given energy. This trajectory grazes the boundary just at the target point $T$. To hit 
the target, the initial velocity $\mathbf{v}_{0}$ of the projectile must have a definite direction (along the bisectrix of the angle $T S Z$ in Fig. 4(b)).

Indeed, the trajectory of the projectile fired to the given target $T$ with the minimal starting speed is a portion of the ellipse passing through $S$ and $T$. One focus of this Keplerian ellipse is located at the center of the Earth, while the second focus belongs to the circle whose center is at the starting point $S$ and whose radius equals $|S Z|$ (see Fig. 4(b)). Therefore this second focus is located at point $F$, at which the segment $S T$ joining the starting point with the target intersects the mentioned circle. Knowing locations of both foci for the elliptic trajectory of the projectile, we can easily find the firing angle with the help of the optical property of the ellipse. Since a light ray emitted from the focus $E$ to $S$ must be reflected at $S$ by an elliptical mirror towards the second focus $F$, the tangent to the ellipse at $S$ (and hence the direction of initial velocity $\mathbf{v}_{0 \text { min }}$ ) is the bisectrix of the angle $F S Z$ (or $T S Z$, which is the same; see Fig. 4(b)).

Any target lying within the bounding surface can be reached by two trajectories of the given family. One of them (the trajectory labeled 1 in Fig. 4(a)) first passes through the target point $T$, and only then grazes the boundary. The other (labeled 2 in Fig. 4(a)) is generated by a projectile launched in a different direction, which is closer to the local vertical line at the starting point. This trajectory first grazes the boundary, and only then passes through the target point.

If we increase the initial speed of the fragments, the bounding surface expands and its shape becomes more spherical. Indeed, as we can see from Eq. (4), the eccentricity $e$ of the bounding ellipse becomes smaller and tends to zero as the initial speed approaches the escape velocity. If the initial speed $v_{0}$ equals the circular velocity $v_{\text {circ }}$ for the initial point, the distance between the foci of the bounding ellipse is one third its major axis. That is, the eccentricity of the ellipse is $1 / 3$ if $v_{0}=v_{\text {circ }}$.

As the initial speed tends to zero, the eccentricity of the envelope approaches unity. Then the apexes (the ends of the major axis) of the bounding ellipse approach its foci, and the ellipse becomes very narrow and stretched, being spanned over the initial point $S$ and the center of the Earth $E$. This limiting case of a degenerate ellipse corresponds to the parabolic shape of the above-mentioned bounding surface for the trajectories of the fragments moving within a restricted spatial region (in the vicinity of the initial point $S$ ) in which the gravitational field can be regarded as uniform.

\section{Useful Applications of the Envelope}

Knowing the boundary surface can be useful in solving various problems concerning the orbital motion. For example, we can easily find the minimal firing speed of a projectile needed to hit a given target from a given starting point. Suppose we have a target $T$ (see Fig. 4(b)) at a given location. Let the target location be determined by distance $r_{T}=|E T|$ from the force center $E$ (the center of the Earth) and distance $l_{T}=|S T|$ from the given starting point $S$ (whose distance from the force center is $r_{0}=|E S|$ ). What is the minimal initial speed and what should be the firing angle?

The firing speed is minimal if the target $T$ lies on the bounding surface. Since this boundary is an ellipse, the sum of distances $|E T|$ and $|S T|$ from $T$ to its foci (points $E$ and $S$ ) is equal to the major axis of the bounding ellipse: $|E T|+|S T|=r_{Z}+r_{N}$. The sum $|E T|+|S T|$ is just the sum of given distances $r_{T}$ and $l_{T}$ to the target from $E$ and $S$. Let us denote this sum as $b: r_{T}+l_{T}=b$. Thus, we can equate this given value $b$ to the major axis $r_{Z}+r_{N}$, which has already been calculated above, when we derived expression (4) for the eccentricity of the bounding ellipse:

$$
b=r_{Z}+r_{N}=r_{0} \frac{v_{\mathrm{esc}}^{2}+v_{0}^{2}}{v_{\mathrm{esc}}^{2}-v_{0}^{2}} .
$$

Solving this equation, Eq. (5), for $v_{0}$, we obtain the desired minimal firing speed:

$$
v_{0 \min }^{2}=v_{\mathrm{esc}}^{2} \frac{b-r_{0}}{b+r_{0}}
$$

Equation (6) shows that for a given position of the starting point $S$ the minimal firing speed depends only on $b$, that is, on the sum of distances $r_{T}$ and $l_{T}$ that determine the target location $\left(b=r_{T}+l_{T}\right)$. According to Eq. (6), the firing speed is zero if $b=r_{0}$, that is, for any target that lies on the segment $S E$ 
joining the starting point and the center of the Earth. The minimal firing speed $v_{0}$ min tends to the escape velocity $v_{\text {esc }}=\sqrt{2 g R^{2} / r_{0}}$ as the target is moved away to infinity $($ as $b \rightarrow \infty)$.

As we have shown above, the firing angle can be easily found with the help of the optical property of the ellipse. Since a light ray emitted from the focus $E$ to $S$ must be reflected at $S$ by an elliptical mirror towards the second focus $F$, the tangent to the ellipse at $S$ (and hence the direction of initial velocity $\mathbf{v}_{0 \text { min }}$ ) is the bisectrix of the angle $F S Z$ or $T S Z$ (see Fig. 4(b)).

\section{Shooting Equatorial Targets from the Pole}

Next we discuss particular examples of trajectories traced by the projectiles launched with minimal initial speeds to given targets.
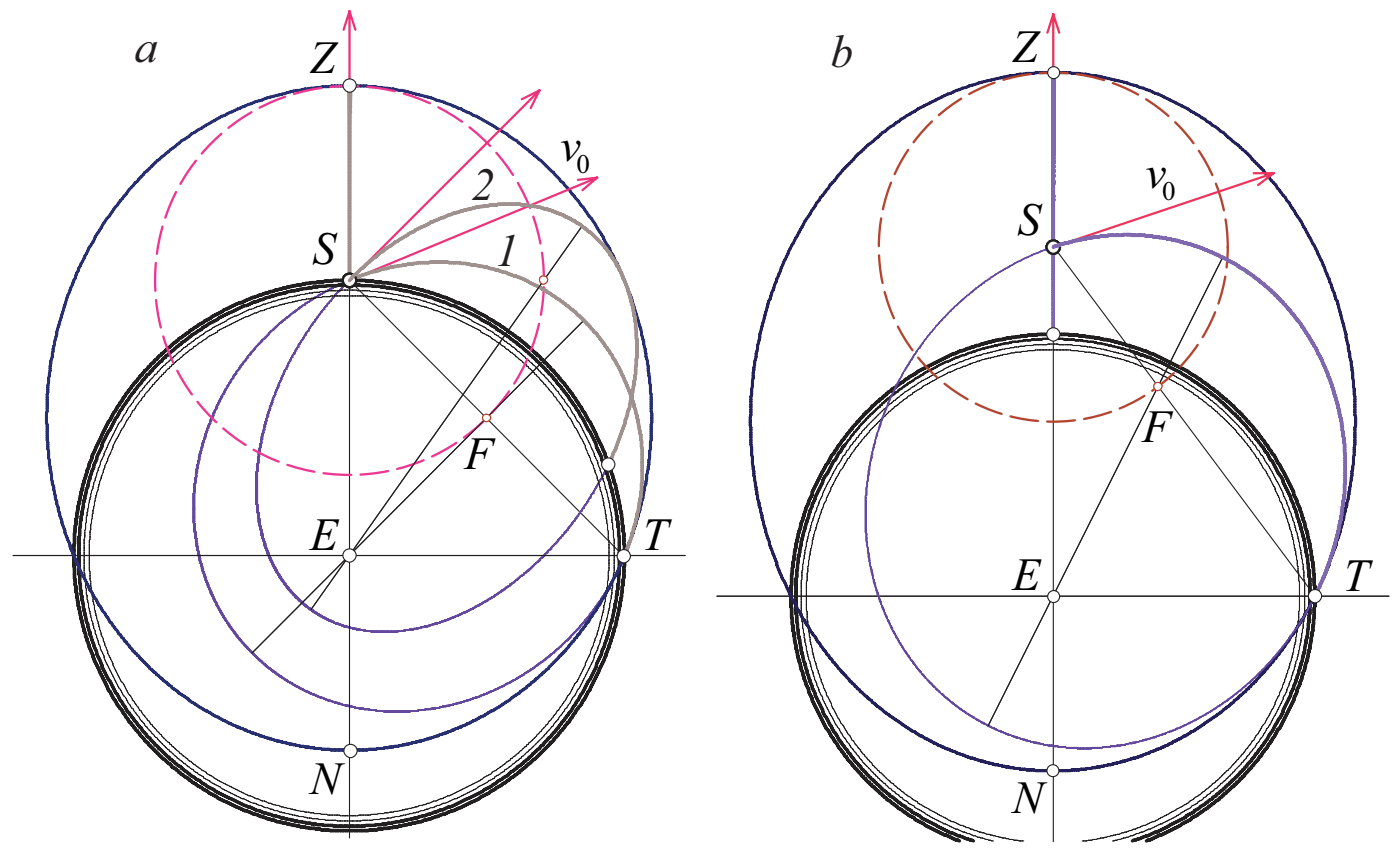

Figure 5: Examples of trajectories that correspond to the minimal starting speed of the projectile for a given starting point $S$ and a given target $T$ : (a) from the North Pole to the Equator; (b) from a starting point $S$ that is raised to the height of $R / 3$ over the Pole, to the Equator.

Let the starting point $S$ be located at the North Pole, and the target $T$ on the Equator (Fig. 5(a)). In this case both points $S$ and $T$ are located on the surface of the Earth, so their distances from the center of the Earth are both equal to the Earth's radius $R: r_{0}=r_{T}=R$. Hence the distance $l_{T}$ of the target $T$ from the starting point $S$ equals $\sqrt{2} R$, so that $b=r_{T}+l_{T}=(1+\sqrt{2}) R$. For the minimal starting speed Eq. (6) yields in this case $v_{0 \text { min }}=0.9102 v_{\text {circ }}$, where $v_{\text {circ }} \approx 7.9 \mathrm{~km} / \mathrm{s}$ is the circular speed for the (hypothetical) orbit whose radius equals the Earth's radius (the 1 st cosmic velocity).

At the target point $T$ both ellipses, the desired trajectory (labeled 1 in Fig. 5(a)) and the envelope bounding surface, have a common tangent. According to the optical property, a ray ET emitted from the common focus $E$ of these ellipses must be reflected at $T$ by both curves toward their second foci ( $F$ and $S$ respectively). Therefore all three points $(T, F$, and $S$ ) lie on the same straight line $T S$.

The angle $T S Z$ (or $F S Z$, which is the same) in this case equals $135^{\circ}$. The initial velocity $\mathbf{v}_{0}$ must be directed along the bisectrix of this angle. Hence the angle between vector $\mathbf{v}_{0}$ and the upward vertical line must equal $67.5^{\circ}$.

It is interesting to compare this trajectory 1 with the trajectory 2 (see Fig. 5(a)), which is traced by the projectile launched with the same initial speed $v_{0}$ at an angle of $45^{\circ}$. We note that on the surface of the 
Earth the range of this projectile is smaller than in the preceding case, in which the projectile is fired with the same starting speed at an angle of $67.5^{\circ}$, contrary to the situation on the "flat Earth."

As another example, we consider a starting point $S$ located at a height of one third the Earth's radius $R$ over the North Pole, with the target still on the Equator (Fig. 5(b)). In this case $r_{0}=\frac{4}{3} R, r_{T}=R$, and $l_{T}=\frac{5}{3} R$, so that $b=\frac{8}{3} R$. For the minimal starting speed in this case Eq. (6) yields $v_{0 \text { min }}^{2}=\frac{2}{3} v_{\text {circ }}^{2}$, or $v_{0 \text { min }}=0.8165 v_{\text {circ }}$. From the triangle $T S E$ we can see that the sine of angle $T S E$ equals $3 / 5$. The initial velocity $\mathbf{v}_{0}$ must be directed along the bisectrix of the angle $T S Z$. Hence the angle between vector $\mathbf{v}_{0}$ and the upward vertical line must equal $71.565^{\circ}$. The trajectory of this projectile is a portion $S T$ of an ellipse whose foci are located at $E$ (the Earth's center) and $F$. The latter point lies on the straight line $S T$ joining the starting point and the target.

The bounding ellipse and the trajectories, shown in Figure 5 and subsequent figures, are generated with the help of the relevant simulation program of the software package [6].

\section{Targets in the Northern and Southern Hemispheres}
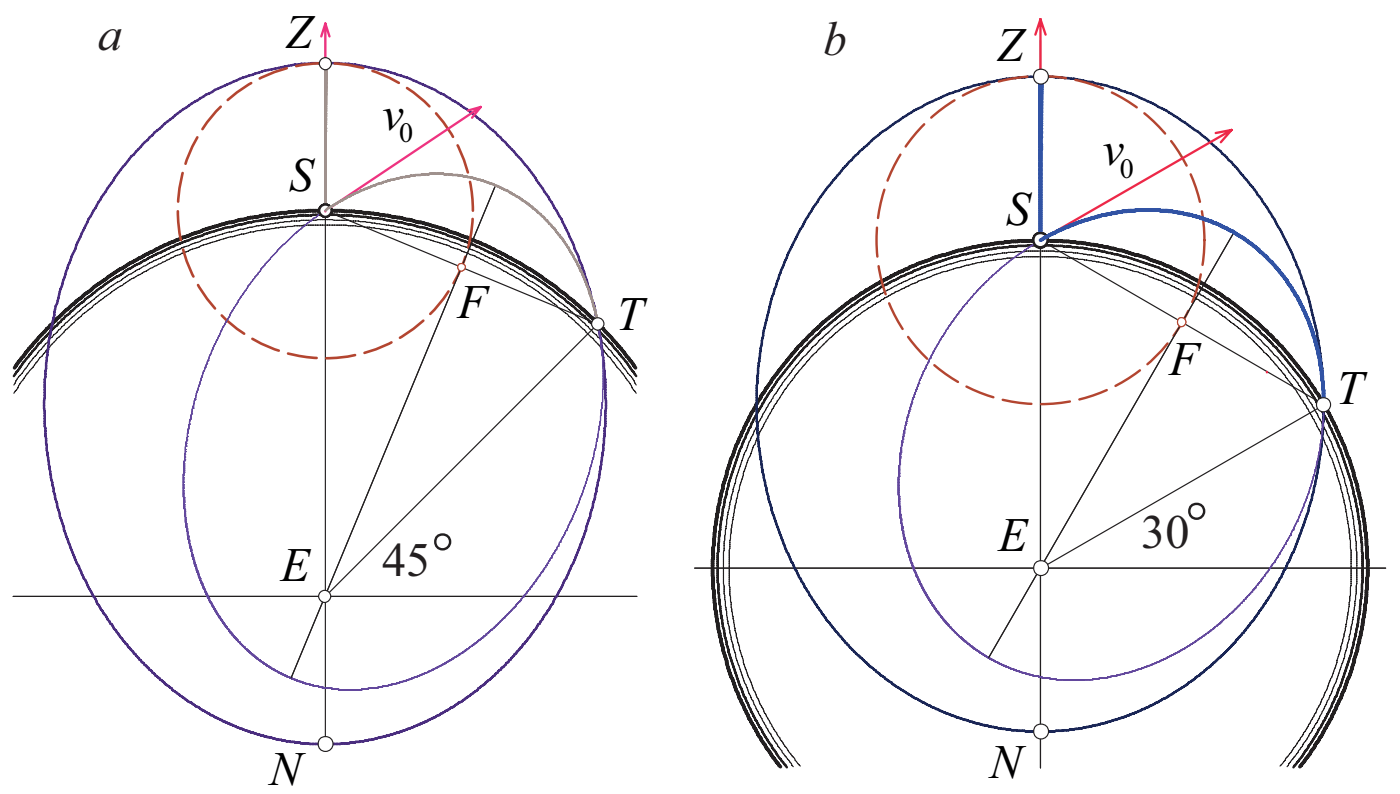

Figure 6: Examples of trajectories that correspond to the minimal starting speed of the projectile for a given starting point $S$ at the North Pole and a given target $T$ : (a) a target located at northern latitude $45^{\circ}$; (b) at northern latitude $30^{\circ}$

Figures 6(a) and 6(b) show trajectories of missiles with minimal shooting speed aimed at targets located at northern latitudes $45^{\circ}$ and $30^{\circ}$, respectively. Again both points $S$ and $T$ are located on the surface of the Earth, so that their distances from the center of the Earth are equal to the Earth's radius $R: r_{0}=r_{T}=R$. For the target $T$ at $45^{\circ}$ latitude the distance $l_{T}$ of the target from the starting point $S$ equals $2 R \sin 22.5^{\circ}$, so that $b=r_{T}+l_{T}=1.765 R$. According to Eq. (6) the minimal starting speed $v_{0 \text { min }}=0.744 v_{\text {circ }}$. The angle $T S Z$ in this case equals $112.5^{\circ}$. The initial velocity $\mathbf{v}_{0}$ must be directed along the bisectrix of this angle. Hence the angle between vector $\mathbf{v}_{0}$ and the upward vertical line must equal $56.25^{\circ}$.

Similarly, for the example in Fig. $6(\mathrm{~b}), r_{0}=r_{T}=R$. For the target $T$ at $30^{\circ}$ latitude the distance $l_{T}$ of the target from the starting point $S$ also equals $R$, so that $b=r_{T}+l_{T}=2 R$. According to Eq. (6), the minimal starting speed is $v_{0 \text { min }}=\sqrt{2 / 3} v_{\text {circ }}=0.8165 v_{\text {circ }}$. The angle $T S Z$ in this case equals $120^{\circ}$. Hence the angle between vector $\mathbf{v}_{0}$ and the upward vertical line must equal $60.0^{\circ}$. 

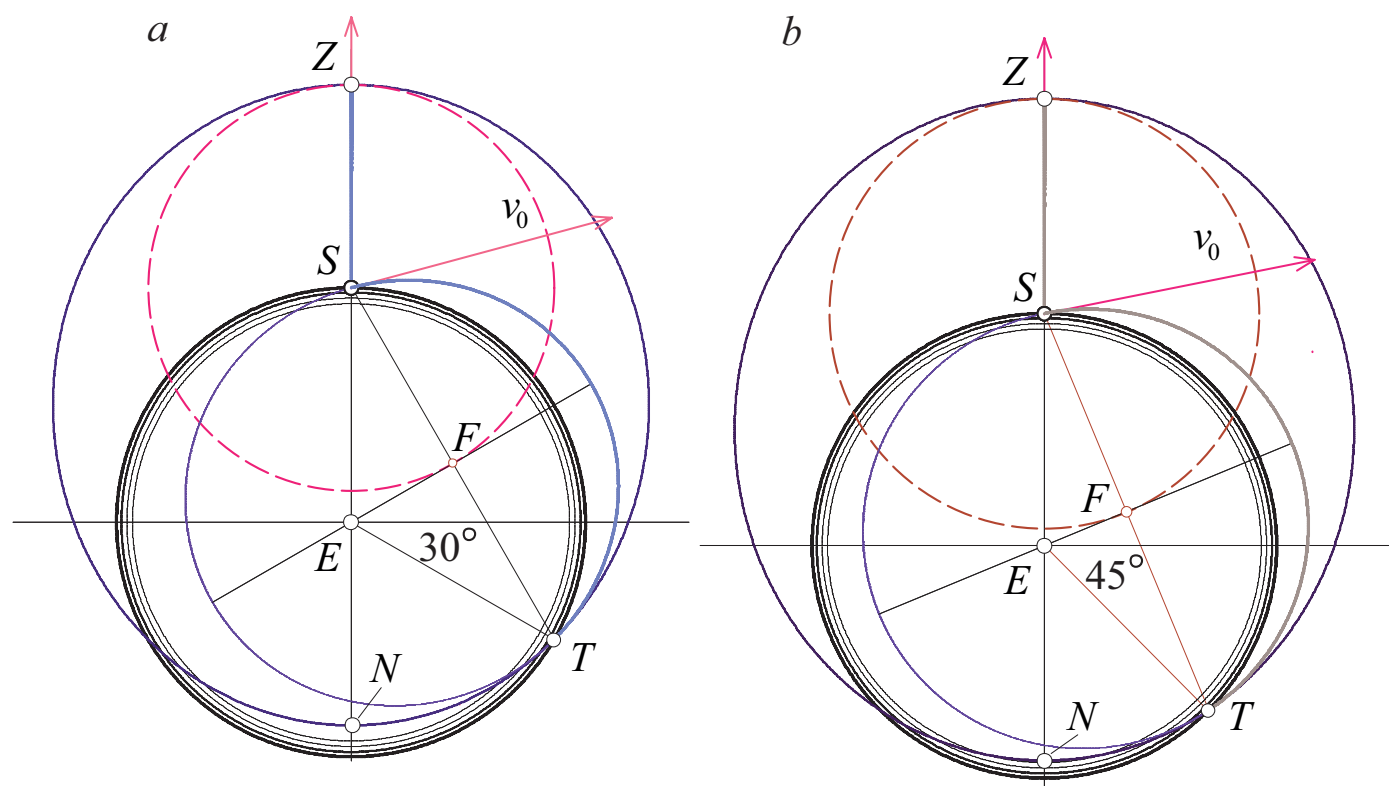

Figure 7: Examples of trajectories that correspond to the minimal starting speed of the projectile for a given starting point $S$ at the North Pole and a given target $T$ : (a) a target located at southern latitude $30^{\circ}$; (b) at southern latitude $45^{\circ}$.

Figures 7(a) and 7(b) show trajectories (and the envelope bounding surfaces) of missiles with minimal shooting speed aimed at targets located at southern latitudes of $30^{\circ}$ and $45^{\circ}$, respectively. We leave the calculation of the required shooting speeds and the firing angles for these cases to the reader. Correctness of the calculations can be verified with the help of an orbit simulation program.[6]

We note that for a starting point located at the North Pole and a target on the surface of the Earth, the minimal initial speed of the missile tends to the circular velocity $v_{\text {circ }} \approx 7.9 \mathrm{~km} / \mathrm{s}$ (the speed of a satellite orbiting the Earth in an extremely low circular orbit) as the target is moved towards the South Pole. In order for a missile launched from one pole to hit a target located at the opposite pole, the shooting speed must be at least a little bit greater than $v_{\text {circ }}$.

\section{Envelope of the Parabolic Trajectories}

Although there exist in the literature[1]-[5] several elegant analytical derivations of the "safety paraboloid" for projectiles moving in a uniform gravitational field, here we present for completeness a simple geometrical proof of this well-known result (see Fig. 1). The derivation is similar to that in Sec. III for the elliptical shape of the envelope of the family of Keplerian ellipses.

All the parabolic trajectories of the family with given initial speed $v_{0}$ have a common starting point $S$ and a common directrix $A A^{\prime}$ (see Fig. 8). The locus of foci of all the parabolas is a circle whose center is located at the starting point $S$, and whose radius is equal to the distance from $S$ to point $Z$, which is the maximum height reached by the projectile launched vertically. This locus is shown by the dashed circle in Fig. 8. Compare it with the circular locus of foci of elliptic orbits in Figs. 2-7.

In all cases, independently of the initial speed imparted to the projectiles, the center of this circle coincides with the starting point $S$, and its radius equals the distance from $S$ to point $Z$. This is also true for the case under consideration, namely, for the limiting case of a very small initial speed $v_{0} \ll v_{\text {circ }}$, in which the upper portions of ellipses near their apexes can be approximately regarded as parabolas (approximation of a "flat Earth").

To prove that the envelope of parabolic trajectories is a parabola, we choose an arbitrary target point $T$ below the line $A A^{\prime}$, and draw a circle with a center at $T$ (see Fig. 9). Let the radius of this circle be equal 


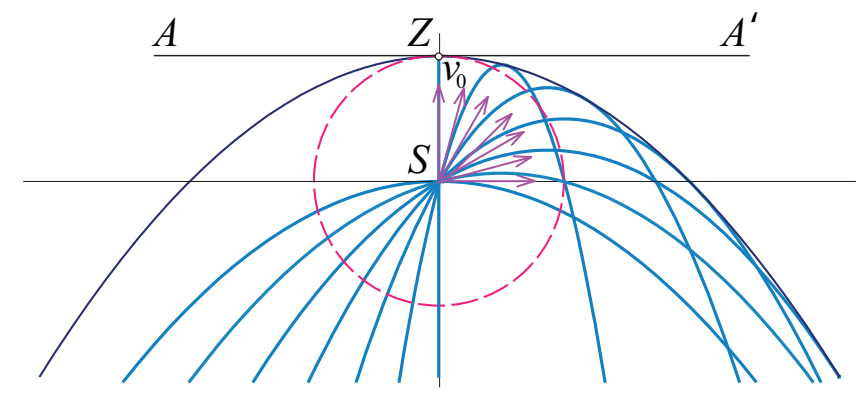

Figure 8: The envelope of the parabolic trajectories traced in a uniform field by projectiles launched from one point with equal speeds, and the locus of foci of these parabolas (dashed circle).

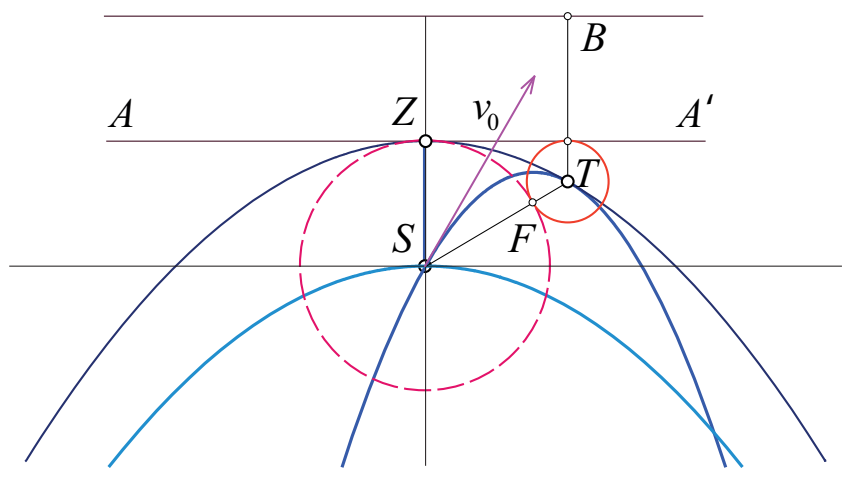

Figure 9: Geometric construction for the proof of the parabolic shape of the "safety domain" boundary (see text for details).

to the distance from $T$ to line $A A^{\prime}$, so that the circle grazes this horizontal line. The focus of the parabolic trajectory that passes through $T$ must belong to this circle, because line $A A^{\prime}$ is the directrix of this parabola. At the same time this focus must lie on the dashed circle (the locus of all the foci). Again we examine three possibilities:

1. The circle centered at $T$ crosses the dashed circle. In this case there exist two foci, and hence two parabolic trajectories of the family pass through the given target $T$.

2. The circle centered at $T$ has no common points with the dashed circle. This means that a trajectory passing through $T$ does not exist: the given target $T$ is out of reach from $S$ for a projectile with the given initial velocity $v_{0}$.

3. Finally, the circle centered at $T$ grazes the dashed circle. In this case there exists a unique trajectory of the family that passes through the given target $T$. This means that in this case the point $T$ belongs to the desired boundary (see Fig. 9). At this point $T$ the trajectory grazes the boundary.

To see that this boundary is a parabola, we draw one more horizontal line whose distance from the starting point $S$ equals twice the upper height $|S Z|$ of the projectile launched vertically (see Fig. 9). In case the two circles graze one another, the distance $|T B|$ from the target $T$ to this line just equals the distance $S T$. This proves that point $T$ belongs to a parabola, whose directrix is the horizontal line passing through $B$, and whose focus is located at the starting point $S$. Thus we have proved that the envelope of parabolic trajectories is a parabola whose apex is point $Z$ and whose focus is point $S$. We also note that this boundary of the "safety domain" can be obtained from the parabolic trajectory of the projectile that launched from $Z$ horizontally, by raising this parabola as a whole through distance $|S Z|$ (see Fig. 9).

The focus $F$ of the parabolic trajectory that passes through $T$ lies on the straight line joining the starting point $S$ and the target $T$ (see Fig. 9). This can be easily seen from the optical property of a parabolic 
mirror, according to which any light ray emitted from the focus is reflected in the direction of the axis of the parabola. Since at point $T$ both parabolas (the trajectory and the envelope) graze each other and have a common tangent, the light rays from their foci ( $F$ and $S$ respectively) are both reflected in the same direction. This means that points $S, F$, and $T$ lie on the same straight line.

The above-mentioned optical property of a parabolic mirror allows us to easily find the required direction of the firing speed $\mathbf{v}_{0}$ at the initial point $S$ in order to hit a given target $T$. Indeed, a light ray directed vertically upward is reflected at point $T$ by the parabolic mirror in the direction of its focus $F$. Hence the tangent to this parabola at point $T$ (as well as the direction of vector $\mathbf{v}_{0}$, Fig. 9) is the bisectrix of the angle $Z S F$ (or $Z S T$ ).

\section{Summary}

In this paper alternative derivations are presented for the boundary of the "safety domain" of elliptic orbits of satellites (and ballistic trajectories of missiles) in a central Newtonian gravitational field, and for parabolic trajectories of projectiles in a uniform gravitational field, when the projectiles are fired from one point with the same initial speed in various directions. The derivations for the shape of the envelope surface are almost entirely based on geometrical properties of ellipses and parabolas. The boundary surface can be useful for solving practical problems of finding the minimal firing speed and the required initial direction of a projectile that is to hit a given target from a given starting point. These applications are illustrated through several examples, and supported by computer simulations.

\section{References}

[1] D. Donnelly, "The parabolic envelope of constant initial speed trajectories," Am. J. Phys. 60, 11491151 (1992).

[2] A.P. French, "The envelopes of some families of fixed-energy trajectories," Am. J. Phys. 61, 805-810 (1993).

[3] A. G. Alenitsyn, E. I. Butikov, A. S. Kondratyev, Concise Handbook of Mathematics and Physics (CRC Press, New York, 1997), pp. 241-242.

[4] M. Baće et al. "The envelope of projectile trajectories," Eur. J. Phys. 23, 637-642 (2002).

[5] E. I. Butikov, "Comment on 'The envelope of projectile trajectories,” Eur. J. Phys. 24, L5-L9 (2003).

[6] E. I. Butikov, Planets and Satellites (Physics Academic Software, American Institute of Physics, 1999). An updated version for the Windows operating system is available as a free download at $<$ http://butikov.faculty.ifmo.ru>.

[7] L. D. Landau and E. M. Lifschitz Mechanics. New York: Pergamon (1976).

[8] Ch. Kittel, W. D. Knight, M. A. Ruderman Mechanics, Berkeley Physics Course, v. 1, New York: McGraw-Hill (1965).

[9] E. I. Butikov, Motions of Celestial Bodies, IOP Publishing Ltd. (2014). 